\title{
Potencial IMPACTO ENERGÉTICO DE LOS VEHÍCUlos ELÉCTRICOS EN La RED DE Distribución DEL Distrito NaCional, República Dominicana
}

\author{
Energy impact of electric vehicles in the distribution grid of national \\ District, Dominican Republic
}

\author{
Edison Antolín Álvarez Jerez , Henry César Caraballo Durána ${ }^{a}$, \\ Robinson Radhamés Gómez Then ${ }^{a}$, Manny Alexander Montero \\ Dilonéa ${ }^{a}$ Israel Josué Soto LeOnora ${ }^{a}$, Elvin Arnaldo Jiménez \\ Matos $^{a}$ y Miguel Euclides Aybar Mejía ${ }^{a}$
}

Cómo citar: Álvarez Jerez, E. A., Caraballo Durán, H. C., Gómez Then, R. R., Montero Diloné, M. A., Soto Leonor, I. J., Jiménez Matos, E. A., \& Aybar Mejía, M. E. (2019). Potencial impacto energético de los vehículos eléctricos en la red de distribución del Distrito Nacional, República Dominicana. Ciencia, Ingenierías y Aplicaciones, 2(1), 45-66. Doi: https://doi.org/10.22206/cyap.2019.v2i1.pp45-66

\section{Resumen}

El mundo se encuentra en una transición energética, lo que trae como consecuencia el surgimiento de tecnologías para contrarrestar la dependencia de los derivados de petróleo en la movilidad vehicular. En respuesta a esto, se presentan los vehiculos eléctricos como una alternativa ecológica para el desplazamiento cotidiano de los seres humanos. Se estableció como objetivo determinar el impacto de conectar y cargar vehiculos eléctricos en la red de distribución, para ello se utilizó la herramienta de simulación Power System Simulator (PSS/ADEPT) y hojas de cálculos, en donde se realizaron corridas de flujos de carga a diferentes escenarios de penetración vehicular, evaluando en cada caso parámetros eléctricos como cargabilidad y caída de tensión en los conductores troncales y transformadores de los circuitos seleccionados. Los resultados arrojados en las simulaciones permiten afirmar que la infraestructura actual de los circuitos seleccionados no está apta para soportar una penetración considerable de vehículos eléctricos, donde en circuitos

\footnotetext{
anstituto Tecnológico de Santo Domingo (INTEC), Ingeniería Eléctrica Correo-e de autores:

edisonaj12@gmail.com (E. Álvarez), henrycaraballogz@gmail.com (H. Caraballo),robinsongomez2697@ gmail.com (R. Gómez), mannymontero0298@gmail.com (M. Montero), israeljosue37@hotmail.com (I. Soto), elvin.jimenez@intec.edu.do (E. Jiménez), miguel.aybar@intec.edu.do (M. Aybar).
} 
como el EMBA-105 a un $10 \%$ de penetración vehicular eléctrica las pérdidas permisibles fueron superadas. Con estos resultados se pudo detectar cuáles circuitos de la red de distribución eléctrica seleccionados pudieran colapsar con la entrada masiva de la movilidad eléctrica.

Palabras clave: vehículos eléctricos; red de distribución; factor de coincidencia; Power System Simulator (PSS / ADEPT); pérdidas técnicas; gases de efecto invernadero; demanda máxima.

\begin{abstract}
The world is currently in a transition of energy, which brings as part of the operation of technologies to counteract the dependence of petroleum derivatives on vehicular mobility. That is the main reason why electric vehicles arise, as an ecological alternative for the daily movement of human beings. The objective of this investigation was to analyze how the integration of these electric vehicles affects energetically the distribution grid of the National District of the Dominican Republic. To determine the impact of connecting and charging electric vehicles in the distribution network, the simulation tool PowerSystem Simulator (PSS / ADEPT) and spreadsheets were used, where load flow runs were performed to different vehicular penetration scenarios, evaluating in each case, electrical parameters such as chargeability and voltage drop in the trunk conductors and transformers of the selected circuits. The results obtained in the simulations show that the current infrastructure of the selected circuits is not suitable to support a considerable penetration of electric vehicles, where in circuits such as the EMBA-105 a 10 $\%$ vehicle penetration the allowable losses were overcome. With these results, conclusions are obtained regarding the socioeconomic impact and environment that this technology represents in this country.
\end{abstract}

Keywords: electric vehicles; distribution grid; coincidence factor; technical losses; greenhouse gases; maximum demand. 


\section{Introducción}

Los vehículos de motor han sido utilizados como el medio de transporte terrestre predilecto a nivel mundial, representando un agente considerable de deterioro progresivo del medio ambiente, ya que su fuente de energía proviene de los combustibles fósiles; más de la mitad de la producción mundial de petróleo se destina al sector del transporte (Transportation, 2015). Sector que desempeña un papel preponderante en la contaminación del aire y en el cambio climático (Alaswad et al., 2016). Existen normas para el control de estas emisiones, en la medida en que avanza la tecnología (Jenn, Azevedo, \& Michalek, 2019), para así disminuir las emisiones producidas por la interacción entre los combustibles fósiles y los parámetros del motor de combustión interna (Wallington, Kaiser, \& Farrell, 2006). Por ejemplo, la Convención Marco de las Naciones Unidas sobre el Cambio Climático plantea un acuerdo generalizado para reducir las emisiones de $\mathrm{CO} 2$ del transporte como mínimo en un $50 \%$ a más tardar para 2050 (Geerlings \& Zuidwijk, 2017).

Los vehículos eléctricos (VE) representan una opción para reducir las emisiones de efecto invernadero del transporte personal (Riesz, Sotiriadis, Ambach, \& Donovan, 2016), además, los VE tienen asociados algunos beneficios para el medio ambiente, tales como la reducción del ruido y la mejora de la calidad del aire (Ferrero, Alessandrini, \& Balanzino, 2016), pero a su vez, los vehículos eléctricos presentan un paradigma en los mercados eléctricos, ya que producen un incremento de la demanda eléctrica y, en algunos puntos de la red, acrecientan las pérdidas eléctricas del sistema.

En el primer semestre del año 2018 se han vendido 700,000 vehículos eléctricos (100\% eléctricos e híbridos) en el mundo, superando el mismo periodo del año 2017 en un $69 \%$. De los vehículos vendidos en este periodo, el $1.6 \%$ son eléctricos y es probable que su participación se eleve en un $2 \%$ al terminar el 2018 (Agudelo, 2018). La Agencia Internacional de Energía (AIE) dice que las ventas de vehículos podrían aumentar en promedio a nivel mundial un $24 \%$ anual hacia el 2030. Estos escenarios 
muestran cómo la matriz de transporte está cambiando hacia fuentes de energía sostenibles (Díaz, 2017). La empresa Turiscar, empresa de vehículos eléctricos para hoteles, se ha dedicado a la importación de vehículos eléctricos en República Dominicana. En el sector de educación, la Universidad Dominicana O\&M realizó un proyecto en el año 1991 de movilidad eléctrica. En el 2017, la Comisión Nacional de Energía (CNE) y la Corporación de Energía Eléctrica de Corea (KEPCO), conjuntamente, dieron la introducción formal a los VE, celebrando seminarios de vehículos eléctricos en la República Dominicana (CNE, 2017). Esto indica que el Estado dominicano se encamina hacia la transformación de la fuente de energía de su matriz de transporte.

Cuando los VE están conectados a una red de distribución eléctrica, el flujo de energía entre los VE y la red eléctrica puede ser bidireccional: un VE en modo de carga es una carga para la red (ElNozahy \& Salama, 2014), mientras que un VE que inyecta potencia es una fuente de energía para la red (Bremermann, Matos, Lopes \& Rosa, 2014). Sin embargo, las arquitecturas de las redes de transmisión y distribución aún se centran en el diseño y las reglas operativas tradicionales (Anastasiadis, Kondylis, Polyzakis \& Vokas, 2019).

El posible desafío para las redes de energía radica en que la penetración de un gran número de VE puede desencadenar oleadas extremas en la demanda en horas punta, y por lo tanto, dañar la estabilidad y la seguridad de las redes de energía existentes (Anastasiadis et al., 2019).

Aquí radica la importancia de realizar un estudio preliminar sobre cómo la penetración de los VE afectará la red de distribución en el Distrito Nacional de la República Dominicana. Actualmente, no se conoce de manera pública ningún estudio en el país, enfocado en los efectos de los VE en la red de distribución nacional.

Para determinar el impacto de los VE en la red de distribución eléctrica dominicana, fue necesario simular cuál es el impacto de los VE en ciertos circuitos de distribución. Los circuitos evaluados pertenecen a la 
distribuidora de electricidad EDESUR de la ciudad de Santo Domingo, debido al acceso a información que esta distribuidora ofrece, además de que la misma cuenta con la plataforma de simulación que permitió desarrollar los escenarios de flujos de carga de los VE en sus redes de distribución concesionadas. La herramienta de simulación utilizada fue Power System Simulator (PSS/ADEPT) y hojas de cálculos. Por consiguiente, se pudo determinar si la infraestructura actual estaría preparada para abastecer la demanda de potencia eléctrica que representaría una cantidad significativa de VE en el Distrito Nacional.

Los resultados presentados en este proyecto se esperan sean de interés para las empresas encargadas de la planificación de la red de distribución eléctrica. Esta iniciativa de investigación constituye un punto de partida para futuras investigaciones relacionadas a los VE y su impacto en la red de distribución de la República Dominicana.

\section{Metodología}

La metodología utilizada en este caso de estudio fue la presentada en un informe desarrollado en Colombia e intitulado Una Propuesta Metodológica para Dimensionar el Impacto de los Vehiculos Eléctricos sobre la Red (Ceballos Delgado, Caicedo Bravo \& Ospina Arango, 2016), puesto que sirve para evaluar el impacto que tendría la carga de los vehículos eléctricos en la red de distribución. Esto se debe a lo complejo de cuantificar la cantidad de eventos durante el proceso de carga y descarga de VE por día; además de la carga asociada y el impacto en la red eléctrica a través de un enfoque determinista, porque implica múltiples factores que involucran el patrón de movilidad completo conductor (Ul-Haq, Cecati \& El-Saadany, 2018). En la tabla 1 se presenta una matriz comparativa de varias metodologías para análisis de los vehículos eléctricos y sus tecnologías en la red de distribución. El modelo propuesto para el caso de los circuitos a evaluar en República Dominicana solo tomará en cuenta la demanda de los circuitos, el comportamiento del mercado, la planificación de la red de distribución y patrones de cargas. 
Tabla 1. Matriz comparativa de estudios que involucraban análisis de los vehículos eléctricos y sus tecnologías en la red de distribución

\begin{tabular}{|c|c|c|c|c|c|c|c|}
\hline 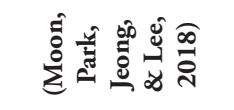 & & * & $\star$ & & $\star$ & $\star$ & \\
\hline 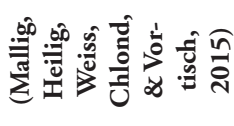 & & $\star$ & & & & * & $\star$ \\
\hline 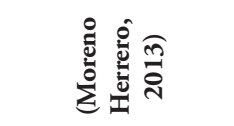 & $\star$ & $\star$ & $\star$ & $\star$ & $\star$ & * & \\
\hline 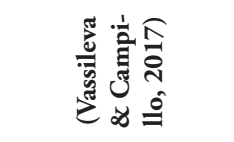 & & & & & & * & $\star$ \\
\hline 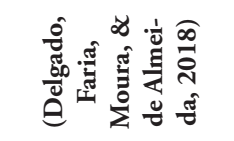 & & $\star$ & $\star$ & & $\star$ & * & $\star$ \\
\hline 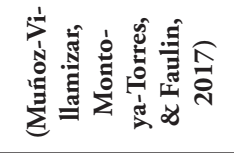 & $\star$ & & & $\star$ & $\star$ & $*$ & \\
\hline 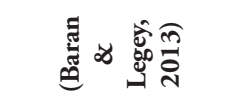 & & * & $\star$ & & & $\star$ & $\star$ \\
\hline 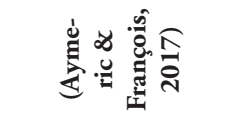 & & $\star$ & & $\star$ & & $\star$ & \\
\hline 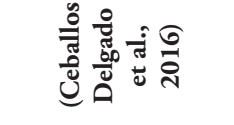 & $\star$ & & & $\star$ & $\star$ & $*$ & $\star$ \\
\hline 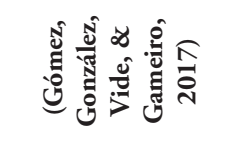 & $\star$ & & $\star$ & & $\star$ & $\star$ & $\star$ \\
\hline 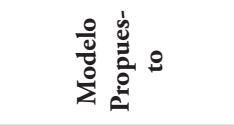 & & * & $\star$ & & & & $\star$ \\
\hline 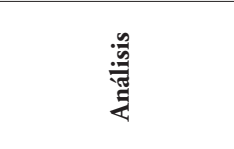 & 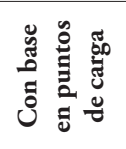 & 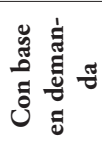 & 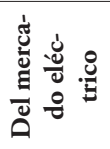 & 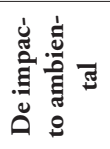 & 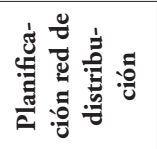 & 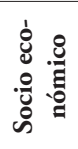 & 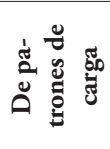 \\
\hline$\dot{z}$ & - & N & $n$ & tr & in & 6 & $\wedge$ \\
\hline
\end{tabular}


Dentro del alcance de la investigación se definió como espacio físico el Distrito Nacional de la República Dominicana, debido a su densidad poblacional (One, 2010) y puesto que en este se encuentran la mayor cantidad de puntos de carga existentes en el país (Electromaps, 2018), un factor a considerar para la ubicación los VE. La investigación no posee un intervalo de tiempo definido, esto es debido a diversos factores, entre los que destaca una limitada adquisición de dichos vehículos por parte de la población, en comparación con los vehículos convencionales; este hecho es corroborado por la Dirección General Aduanas cuando afirma que en el 2017 solo ingresaron al país 28 vehículos eléctricos y para el 2018 el número aumentó a 39 (DGA, 2018).

\section{Variables}

Para poder tener una idea del impacto de los vehículos eléctricos en los circuitos eléctricos a estudiar, es necesario poder estimar su comportamiento al interactuar con las redes eléctricas de distribución y de las cargas eléctricas acopladas a estas redes. Para ello es preciso simular las redes de los circuitos de interés definiendo sus componentes y características propias de cada circuito.

Estas son las siguientes variables concernientes al proyecto en cuestión:

- Factor de demanda de los circuitos.

- Curva de demanda de los circuitos.

- Tasa de crecimiento de carga por circuito.

- Costo de la energía por circuito.

- Potencia demandada por vehículo eléctrico.

- Topología de los circuitos.

- Cantidad de vehículos por circuito.

- Potencia por kilómetro de red de los distintos circuitos.

- Energía vendida a los clientes para cada circuito.

- Energía retirada del Mercado Eléctrico Mayorista (MEM) para cada circuito. 
Estas son las variables dependientes tomadas en cuenta en la simulación:

- Pérdidas en los circuitos a analizar.

- Caída de voltaje en los nodos.

- Potencia máxima demandada en los diferentes casos y porcentaje de congestión en líneas de distribución.

- Factor de coincidencia.

- Ampacidad en los conductores troncales.

- Costo de la energía consumida por circuito.

- Impacto en el mercado eléctrico dominicano.

\section{Simulación}

Para la etapa de simulación se definieron dos escenarios: uno, sin la incorporación de VE, cuyo caso de estudio se centra en un flujo de carga de la red de distribución del circuito sin la carga representativa de los $\mathrm{VE}$, es decir, en situación actual. Otro, que consiste en la incorporación de VE al circuito de interés; dicha penetración se añadió al circuito en forma de carga representativa de los VE. De tal forma el escenario se subdivide en seis casos de estudios, centrados en realizar un flujo de carga de la red de distribución de circuitos con la inserción de un $10 \%, 20 \%$, $40 \%, 60 \%, 80 \%, 100 \%$, de la población vehicular del circuito, siendo sustituida por VE y su respectiva carga a la red.

Para determinar esta carga vehicular se utilizó la información propuesta por la Oficina Nacional de Estadísticas (ONE) en el rx censo de población y vivienda realizado en el 2010, donde se obtuvo información sobre la cantidad vehicular de uso privado de los diferentes sectores que componen el Distrito Nacional (One, 2010). Una vez obtenida esta información sobre la cantidad de automóviles por año, se realizó un ajuste de la tendencia para encontrar el polinomio representativo de la curva de crecimiento vehicular y, realizando una extrapolación hacia el 2018, fue posible encontrar la cantidad estimada de vehículos para el 2018 según datos de la Oficina Nacional de Estadística. Una vez obtenida la pendiente de crecimiento de los automóviles desde el 2010 hasta el 2016, se obtuvo como resultado un factor de crecimiento de 1.498096 el cual permite extrapolar la cantidad vehicular de los circuitos para el 2018. 
La cantidad de vehículos convencionales de combustión interna por circuito se muestra en la tabla 2. Con estos datos se estimó la cantidad vehicular que pudieran estar en los circuitos de distribución eléctrica de EDESUR, como son: AHON101, AHON102, AHON103, EMBA101, EMBA103, EMBA105 para el 2010.

\section{Caracterización}

Para la selección de los circuitos se consideró las zonas donde residen los propietarios de este tipo de vehículo eléctrico, según la encuesta realizada a los propietarios de los mismos; además, también se tomó en cuenta que para los circuitos seleccionados EDESUR contará con la base de datos técnicas de las líneas de distribución y perfil de carga en el software PSS/ADEPT.

Tabla 2. Vehículos convencionales por circuito

\begin{tabular}{|c|c|c|}
\hline No. & Circuito & Vehículos Totales \\
\hline 1 & AHON-101 & 5077 \\
\hline 2 & AHON-102 & 1344 \\
\hline 3 & AHON-103 & 4256 \\
\hline 4 & EMBA-101 & 2836 \\
\hline 5 & EMBA-103 & 2836 \\
\hline 6 & EMBA-105 & 5378 \\
\hline
\end{tabular}

Los circuitos seleccionados para la investigación, en todos los casos, tienen un voltaje entre fases de $12.47 \mathrm{kV}$. Para este nivel de tensión, la regulación de tensión máxima permisiva según el Reglamento de Diseño y Construcción de Redes de Distribución Aérea establecido por la Superintendencia de Electricidad SIE-029-2015-MEMI es de $3 \%$ (SIE, 2015). 
En la tabla 3 se presentan los parámetros actuales de los circuitos seleccionados. El alimentador troncal para el circuito EMBA-103 es del tipo $590 \mathrm{kcmil}$ AAAC y tienen una ampacidad de 650 A (Southwire, 2012). Mientras tanto, el resto de circuitos seleccionados en la investigación es del tipo 477 kcmil ACSR, su ampacidad es de 655A (Cable, 2013).

Las pérdidas técnicas que se muestran en la tabla 3 son las pérdidas producidas por Efecto Joule $\left(P_{p e r}=I^{2} R\right)$. El porciento de pérdidas se calcula a partir de la fórmula siguiente (Espinosa y Lara, 1990):

$$
\% P=\frac{P_{p e r}}{P_{T}} \times 100 \%
$$

donde:

$\% P$ : Porciento de pérdidas técnicas

$P_{p e r}:$ Potencia pérdida por Efecto Joule $(\mathrm{kW})$

$P_{T}$ : Potencia total transportada $(\mathrm{kW})$

La corriente de los alimentadores troncales resulta en función de la potencia transportada y el nivel de tensión de la red. Se calcula a partir de la fórmula que se muestra a continuación (Espinosa y Lara, 1990):

$$
I=\frac{S}{3 * V_{L N}}
$$

donde:

I: Intensidad de corriente (A)

$P$ : Potencia demandada (kVA) $V_{L N}:$ Voltaje de línea a neutro $(\mathrm{kV})$

Hay que considerar que las pérdidas máximas para estos circuitos son de $2.5 \%$ 
Tabla 3. Parámetros actuales de los circuitos seleccionados de Edesur

\begin{tabular}{|c|c|c|c|c|c|c|c|c|c|}
\hline Circuito & $\begin{array}{c}\text { Capa- } \\
\text { cidad } \\
\text { Instalada } \\
\text { (MVA) }\end{array}$ & $\begin{array}{c}\text { De- } \\
\text { manda } \\
\text { Máxima } \\
\text { (MVA) }\end{array}$ & $\begin{array}{c}\text { De- } \\
\text { manda } \\
\text { circuito } \\
\text { (MVA) }\end{array}$ & $\begin{array}{c}\text { Caída } \\
\text { de } \\
\text { ten- } \\
\text { sión } \\
\text { (\%) }\end{array}$ & $\begin{array}{c}\text { Pérdi- } \\
\text { das téc- } \\
\text { nicas } \\
\text { (kW) }\end{array}$ & $\begin{array}{c}\text { Pérdi- } \\
\text { das } \%\end{array}$ & $\begin{array}{c}\text { Ampe- } \\
\text { raje } \\
\text { ra de } \\
\text { co- } \\
\text { rirente } \\
\text { (\%) }\end{array}$ & $\begin{array}{c}\text { Conduc- } \\
\text { tor área } \\
\text { kcmil }\end{array}$ \\
\hline $\begin{array}{c}\text { AHON- } \\
101\end{array}$ & 30 & 15.41 & 6.54 & 4 & 67.6 & 1.07 & 303.6 & 52.6 & $\begin{array}{c}477 \\
\text { ACSR }\end{array}$ \\
\hline $\begin{array}{c}\text { AHON- } \\
102\end{array}$ & 30 & 15.41 & 9.47 & 5.07 & 136.4 & 1.47 & 439.5 & 31.4 & $\begin{array}{c}477 \\
\text { ACSR }\end{array}$ \\
\hline $\begin{array}{c}\text { AHON- } \\
103\end{array}$ & 26 & 18.44 & 10.82 & 3.33 & 194.61 & 1.82 & 500.93 & 52.6 & 477 \\
\hline $\begin{array}{c}\text { EMBA- } \\
101\end{array}$ & 50 & 40.96 & 9.92 & 2.67 & 122.83 & 1.29 & 439.5 & 31.4 & $\begin{array}{c}477 \\
\text { ACSR }\end{array}$ \\
\hline $\begin{array}{c}\text { EMBA- } \\
103\end{array}$ & 50 & 31.25 & 5.37 & 2.6 & 52.71 & 1.02 & 248.61 & 53.4 & 465.4 \\
\hline $\begin{array}{c}\text { EMBA- } \\
105\end{array}$ & 50 & 40.96 & 12.89 & 7.42 & 304.49 & 2.46 & 596.76 & 31.4 & $\begin{array}{c}477 \\
\text { ACSR }\end{array}$ \\
\hline
\end{tabular}

Fuente: base de datos de circuitos de EDESUR Dominicana en el periodo del 2018.

Para las simulaciones es necesario conocer el factor de coincidencia, debido a que la carga de los vehículos eléctricos en un circuito no necesariamente ocurre en los mismos intervalos de tiempo, tomando en cuenta, incluso, las tendencias de horarios de cargas de dichos vehículos. Basado en una investigación realizada en Estocolmo, Suecia, sobre el correcto planeamiento de las redes eléctricas para una plataforma de carga de vehículos eléctricos se tomó un factor de 0.67 (Gode, 2016).

La potencia del cargador tomada fue de $3.60 \mathrm{~kW}$ debido a dos factores importantes: el primero, bajo el esquema tarifario dominicano la tarifa de baja tensión simple (BTS1), es decir, la tarifa aplicada a usuarios residenciales no puede exceder los $10 \mathrm{~kW}$, de excederlo el cliente pasa a otra tarifa donde se ve reflejado el costo de la potencia demandada, dicha tarifa es la tarifa con potencia máxima (BTD) (SIE, 2002). El segundo, las instalaciones eléctricas residenciales se realizan para niveles de tensión de $120 \mathrm{~V}$ a $220 \mathrm{~V}$ donde el calibre de mayor ampacidad utilizado para uso interno del hogar es el conductor de calibre ${ }^{\circ} .10$ AWG para instalaciones eléctricas residenciales y el cual posee una corriente permisible de 30 a 35 amperes, limitando la potencia del cargador con base en la tensión utilizada y la sección transversal del conductor (SIE, 2016). 
Al realizar el análisis técnico, para fines económicos, se estará valorizando la energía y así poder evaluar una estimación de flujo de efectivo basado en costo de la energía activa para los circuitos seleccionados. Solo se tomará en cuenta la compra de energía por medio de contratos y energía del mercado Spot en el periodo que comprende entre las 8:00 PM y 12:00 AM.

\section{Valor de energía}

La compra de energía en cada uno de los mercados se valorizará en función de un monto promedio de datos recopilados del último año.

- Mercado de contratos

Para la valorización de la energía del mercado de contratos se utilizó el precio medio de la compra de energía de contratos en el periodo enero - septiembre 2018. Este precio medio es igual a 11.22 U\$Cents/kWh (EDESUR, 2018). Con una tasa de cambio de $50.43 \mathrm{RD} \$ / \mathrm{U} \$$ (Banco Central, 2018) y siendo esta un promedio para compra y venta de divisas del Banco Central de la República Dominicana durante el mes de diciembre 2018, se obtiene un precio medio de 5659.11 RD\$/MWh (Edesur Dominicana, 2018), representando el $68 \%$ de las compras de energía de Edesur.

- Mercado Spot

Para la valorización de la energía del mercado spot se tomó el promedio de los costos marginales declarados por el Organismo Coordinador del Sistema Eléctrico Nacional Interconectado (OC-SENI) en el rango de periodos de 21 a 24; entre diciembre 2017 y noviembre 2018 fue de 6,138.13 RD\$/MWh (EDESUR, 2018), representando el $32 \%$ de las compras de energía de Edesur.

Según el Organismo Coordinador del SENI, las transacciones económicas de energía representan el $65 \%$ del flujo de dinero de todas las transacciones económicas del sistema eléctrico, para poder evaluar todas las transacciones, el valor obtenido de las transacciones de energía se dividirá entre 0.65 , para así poder estimar el $100 \%$ de dichas facturaciones. 
Según la Corporación Dominicana de Empresas Eléctricas Estatales (CDEEE), la tarifa promedio para el caso residencial de la energía eléctrica es de 19 centavos de dólar el kWh (NREL, 2015), que al llevarlo a pesos nos da un valor de 9,581.70 RD\$/MWh para fines de cobro. El nivel de cobranza es cerca de la unidad en estos circuitos, por lo tanto, no se considerarán en el cálculo. Las pérdidas no técnicas se calcularon restando las pérdidas de la simulación del caso actual de las pérdidas totales reportadas por la CDEEE (CDEEE, 2018), que se definen en la tabla 4.

Tabla 4. Parámetros actuales de circuito

\begin{tabular}{|c|c|c|}
\hline No. & Circuito & Pérdidas no técnicas \% \\
\hline 1 & AHON-101 & 21.22 \\
\hline 2 & AHON-102 & 3.67 \\
\hline 3 & AHON-103 & 9.17 \\
\hline 4 & EMBA-101 & 8.49 \\
\hline 5 & EMBA-103 & 4.87 \\
\hline 6 & EMBA-105 & 12.11 \\
\hline
\end{tabular}

Fuente: información suministrada de la base de datos de circuitos de EDESUR Dominicana en el periodo del 2018.

\section{Resultados}

En la simulación se analizaron las siguientes variables: demanda máxima al transformador de potencia asociado al circuito (MVA), demanda individual del circuito (MVA), pérdidas técnicas (\%), caída de tensión (\%) e intensidad de corriente (A) en el alimentador de la red. Los resultados obtenidos para los distintos escenarios de penetración de vehículos eléctrico en cada circuito seleccionado se muestran a continuación (tabla 5 a tabla 10$)$. 
Tabla 5. Impacto en variables dependientes en AHON-101

\begin{tabular}{|c|c|c|c|c|c|c|c|}
\hline $\begin{array}{c}\text { Penetración } \\
\text { VE }\end{array}$ & $\begin{array}{c}\text { Demanda } \\
\text { Max. } \\
(\mathbf{M V A})\end{array}$ & $\begin{array}{c}\text { Demanda } \\
\text { circuito } \\
(\mathbf{M V A})\end{array}$ & $\begin{array}{c}\text { Caída de } \\
\text { tensión } \\
\mathbf{( \% )}\end{array}$ & $\begin{array}{c}\text { Pérdidas } \\
\text { técnicas } \\
\mathbf{( k W )}\end{array}$ & $\begin{array}{c}\text { Pérdidas } \\
\text { Eléctricas } \\
\%\end{array}$ & AMP & $\begin{array}{c}\text { Holgura de } \\
\text { corriente } \\
\mathbf{( \% )}\end{array}$ \\
\hline $0 \%$ & 15.41 & 6.54 & 4 & 67.6 & 1.07 & 303.6 & 52.6 \\
\hline $10 \%$ & 16.41 & 7.8 & 4.8 & 96.64 & 1.28 & 362.31 & 43.5 \\
\hline $20 \%$ & 19.23 & 9.08 & 5.62 & 131.22 & 1.49 & 420.43 & 34.31 \\
\hline $40 \%$ & 21.64 & 11.66 & 7.31 & 217.67 & 1.93 & 540.9 & 15.5 \\
\hline $60 \%$ & 25.78 & 14.28 & 9.07 & 328.46 & 2.39 & 661.11 & -3.3 \\
\hline $80 \%$ & 28.03 & 16.94 & 10.91 & 465.35 & 2.86 & 784.26 & -22.54 \\
\hline $100 \%$ & 31.3 & 19.65 & 12.83 & 630.39 & 3.35 & 919.94 & -43.74 \\
\hline
\end{tabular}

Tabla 6. Impacto en variables dependientes en AHON-102

\begin{tabular}{|c|c|c|c|c|c|c|c|}
\hline $\begin{array}{c}\text { Penetración } \\
\text { VE }\end{array}$ & $\begin{array}{c}\text { Demanda } \\
\text { Max. } \\
\text { (MVA) }\end{array}$ & $\begin{array}{c}\text { Demanda } \\
\text { circuito } \\
(\mathbf{M V A})\end{array}$ & $\begin{array}{c}\text { Caída de } \\
\text { tensión } \\
\mathbf{( \% )}\end{array}$ & $\begin{array}{c}\text { Pérdidas } \\
\text { técnicas } \\
\mathbf{( k W )}\end{array}$ & $\begin{array}{c}\text { Pérdidas } \\
\text { Eléctricas } \\
\mathbf{\%}\end{array}$ & AMP & $\begin{array}{c}\text { Holgura de } \\
\text { corriente } \\
\mathbf{( \% )}\end{array}$ \\
\hline $0 \%$ & 15.41 & 9.47 & 5.07 & 136.4 & 1.47 & 439.5 & 31.4 \\
\hline $10 \%$ & 16.41 & 9.81 & 5.25 & 146.34 & 1.52 & 455.14 & 28.9 \\
\hline $20 \%$ & 19.23 & 10.15 & 5.44 & 156.65 & 1.57 & 469.76 & 26.6 \\
\hline $40 \%$ & 21.64 & 10.82 & 5.82 & 178.39 & 1.68 & 502.1 & 21.6 \\
\hline $60 \%$ & 25.78 & 11.5 & 8.84 & 201.63 & 1.79 & 532.41 & 16.81 \\
\hline $80 \%$ & 28.03 & 12.18 & 9.22 & 226.4 & 1.9 & 563.89 & 11.89 \\
\hline $100 \%$ & 31.3 & 12.86 & 9.97 & 252.7 & 2 & 596.5 & 6.8 \\
\hline
\end{tabular}

Tabla 7. Impacto en variables dependientes en AHON-103

\begin{tabular}{|c|c|c|c|c|c|c|c|}
\hline $\begin{array}{c}\text { Penetración } \\
\text { VE }\end{array}$ & $\begin{array}{c}\text { Demanda } \\
\text { Max. } \\
(\mathbf{M V A})\end{array}$ & $\begin{array}{c}\text { Demanda } \\
\text { circuito } \\
(\mathbf{M V A})\end{array}$ & $\begin{array}{c}\text { Caída de } \\
\text { tensión } \\
\mathbf{( \% )}\end{array}$ & $\begin{array}{c}\text { Pérdidas } \\
\text { técnicas } \\
\mathbf{( k W )}\end{array}$ & $\begin{array}{c}\text { Pérdidas } \\
\text { Eléctricas } \\
\%\end{array}$ & AMP & $\begin{array}{c}\text { Holgura de } \\
\text { corriente } \\
\mathbf{( \% )}\end{array}$ \\
\hline $0 \%$ & 18.44 & 10.82 & 3.33 & 194.61 & 1.82 & 500.93 & 21.73 \\
\hline $10 \%$ & 19.5 & 11.9 & 3.68 & 235.51 & 2.01 & 550.93 & 13.7 \\
\hline $20 \%$ & 20.57 & 12.99 & 4.03 & 280.58 & 2.19 & 601.48 & 6.02 \\
\hline $40 \%$ & 22.73 & 15.19 & 4.75 & 383.6 & 2.55 & 704 & -10 \\
\hline $60 \%$ & 24.91 & 17.41 & 5.48 & 496.41 & 2.81 & 806.02 & -25.94 \\
\hline $80 \%$ & 27.12 & 19.66 & 6.24 & 643.57 & 3.34 & 910.19 & -42.22 \\
\hline $100 \%$ & 29.36 & 21.93 & 7.02 & 802.1 & 3.74 & 1026.69 & -60.42 \\
\hline
\end{tabular}


Tabla 8. Impacto en variables dependientes en EMBA-101

\begin{tabular}{|c|c|c|c|c|c|c|c|}
\hline $\begin{array}{c}\text { Penetración } \\
\text { VE }\end{array}$ & $\begin{array}{c}\text { Demanda } \\
\text { Max. } \\
\text { (MVA) }\end{array}$ & $\begin{array}{c}\text { Demanda } \\
\text { circuito } \\
(\mathbf{M V A})\end{array}$ & $\begin{array}{c}\text { Caída de } \\
\text { tensión } \\
\mathbf{( \% )}\end{array}$ & $\begin{array}{c}\text { Pérdidas } \\
\text { técnicas } \\
\mathbf{( k W )}\end{array}$ & $\begin{array}{c}\text { Pérdidas } \\
\text { Eléctricas } \\
\mathbf{\%}\end{array}$ & AMP & $\begin{array}{c}\text { Holgura de } \\
\text { corriente } \\
\mathbf{\%})\end{array}$ \\
\hline $0 \%$ & 40.96 & 9.92 & 2.67 & 122.83 & 1.29 & 439.5 & 31.4 \\
\hline $10 \%$ & 42.95 & 10.63 & 2.86 & 141.11 & 1.38 & 492.9 & 23 \\
\hline $20 \%$ & 44.95 & 11.34 & 3.06 & 160.72 & 1.48 & 524.91 & 17.98 \\
\hline $40 \%$ & 49 & 12.77 & 3.45 & 204 & 1.67 & 592.1 & 7.5 \\
\hline $60 \%$ & 53.12 & 14.21 & 3.85 & 252.79 & 1.86 & 657.87 & -2.79 \\
\hline $80 \%$ & 57.32 & 15.65 & 4.26 & 307.18 & 2.05 & 724.54 & -13.21 \\
\hline $100 \%$ & 61.6 & 17.11 & 4.66 & 367.31 & 2.24 & 801.03 & -25.16 \\
\hline
\end{tabular}

Tabla 9. Impacto en variables dependientes en EMBA-103

\begin{tabular}{|c|c|c|c|c|c|c|c|}
\hline $\begin{array}{c}\text { Penetración } \\
\text { VE }\end{array}$ & $\begin{array}{c}\text { Demanda } \\
\text { Max. } \\
\text { (MVA) }\end{array}$ & $\begin{array}{c}\text { Demanda } \\
\text { circuito } \\
(\mathbf{M V A})\end{array}$ & $\begin{array}{c}\text { Caída de } \\
\text { tensión } \\
\mathbf{( \% )}\end{array}$ & $\begin{array}{c}\text { Pérdidas } \\
\text { técnicas } \\
\mathbf{( k W )}\end{array}$ & $\begin{array}{c}\text { Pérdidas } \\
\text { Eléctricas } \\
\%\end{array}$ & AMP & $\begin{array}{c}\text { Holgura de } \\
\text { corriente } \\
\mathbf{( \% )}\end{array}$ \\
\hline $0 \%$ & 31.25 & 5.37 & 2.6 & 52.71 & 1.02 & 248.61 & 53.4 \\
\hline $10 \%$ & 31.96 & 6.08 & 2.95 & 67.5 & 1.16 & 281.48 & 56.7 \\
\hline $20 \%$ & 32.67 & 6.79 & 3.31 & 84.19 & 1.3 & 314.12 & 51.67 \\
\hline $40 \%$ & 34.1 & 8.21 & 4.02 & 123.41 & 1.57 & 371.2 & 41.4 \\
\hline $60 \%$ & 35.55 & 9.65 & 4.76 & 170.62 & 1.85 & 446.76 & 31.27 \\
\hline $80 \%$ & 37 & 11.1 & 5.5 & 226.06 & 2.14 & 513.89 & 19.7 \\
\hline $100 \%$ & 38.48 & 12.57 & 6.27 & 290 & 2.42 & 588.48 & 9.46 \\
\hline
\end{tabular}

Tabla 10. Impacto en variables dependientes en EMBA-105

\begin{tabular}{|c|c|c|c|c|c|c|c|}
\hline $\begin{array}{c}\text { Penetración } \\
\text { VE }\end{array}$ & $\begin{array}{c}\text { Demanda } \\
\text { Max. } \\
\text { (MVA) }\end{array}$ & $\begin{array}{c}\text { Demanda } \\
\text { circuito } \\
(\mathbf{M V A})\end{array}$ & $\begin{array}{c}\text { Caída de } \\
\text { tensión } \\
\mathbf{( \% )}\end{array}$ & $\begin{array}{c}\text { Pérdidas } \\
\text { técnicas } \\
\mathbf{( k W )}\end{array}$ & $\begin{array}{c}\text { Pérdidas } \\
\text { Eléctricas } \\
\%\end{array}$ & AMP & $\begin{array}{c}\text { Holgura de } \\
\text { corriente } \\
\mathbf{\%})\end{array}$ \\
\hline $0 \%$ & 40.96 & 12.89 & 7.42 & 304.49 & 2.46 & 596.76 & 31.4 \\
\hline $10 \%$ & 42.95 & 14.24 & 8.26 & 372.36 & 2.73 & 659.2 & -3.2 \\
\hline $20 \%$ & 44.95 & 15.61 & 9.12 & 447.96 & 3 & 722.67 & -12.92 \\
\hline $40 \%$ & 49 & 18.39 & 10.92 & 623.55 & 3.56 & 852.4 & -33.2 \\
\hline $60 \%$ & 53.12 & 21.31 & 12.82 & 833.92 & 4.14 & 986.57 & -54.15 \\
\hline $80 \%$ & 57.32 & 24.14 & 14.86 & 1082.34 & 4.75 & 1117.59 & -74.62 \\
\hline $100 \%$ & 61.6 & 27.31 & 17.05 & 1372.8 & 5.38 & 1278.56 & -99.77 \\
\hline
\end{tabular}


En la figura 1 se presenta la valorización de las pérdidas eléctricas bajo las condiciones técnicas de los circuitos seleccionados a medida que se incrementa el porciento de penetración de VE.

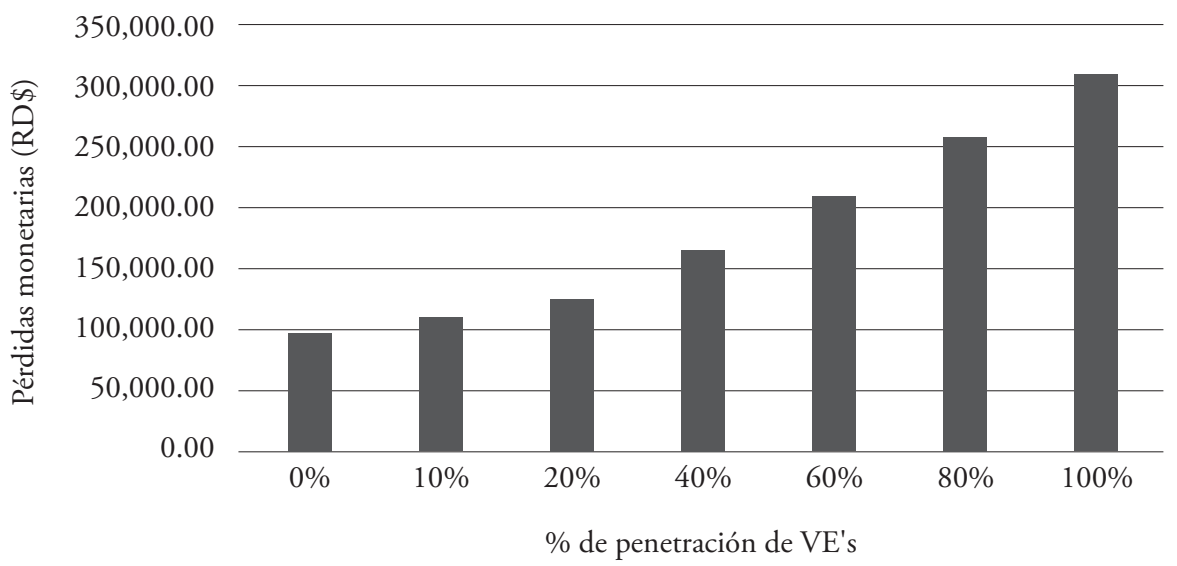

Figura 1. Valorización de la energía comprada y vendida en caso actual.

\section{Discusión de los resultados}

Ante una inserción masiva de VE en las redes del Distrito Nacional se producirían efectos radicales en todas las variables analizadas en esta investigación. La demanda máxima que se tendría en los transformadores de potencia provocaría que estos trabajaran muy cerca de su potencia nominal, y en otros casos, la demanda superaría la capacidad del transformador.

Debido a las penetraciones porcentuales de los VE en la red de distribución y el aumento de demanda que estos conllevan, es visible que existe una disminución en los niveles de tensión de los circuitos analizados, lo que se traduce en un incremento en la caída de tensión desde el conductor troncal hasta el último circuito ramal en análisis. La caída de tensión en condiciones actuales no se encuentra dentro de los parámetros de diseño establecidos por la SIE para redes de distribución de este tipo. Al incrementar la demanda con VE la situación se agudiza alcanzando en algunos de los casos analizados valores superiores al $16 \%$ de caída de tensión. 
Las pérdidas técnicas superan el valor establecido en los circuitos, mayor al $2.5 \%$ establecido por la SIE en el Reglamento de Diseño y Construcción de Redes de Distribución eléctrica aérea. En el caso más crítico supera el $5 \%$ de la potencia activa total transportada.

La corriente eléctrica en el alimentador troncal es una de las variables más críticas, debido a que si el conductor no tiene la capacidad de transportar la corriente demandada puede producir fallas en el mismo. Según los resultados obtenidos en el caso más crítico analizado en este estudio la corriente que circuló por el alimentador del circuito supera los $1200 \mathrm{~A}$ cuando apenas la ampacidad de este es de 640 A.

Tomando en cuenta la compra de energía del mercado spot y el mercado de contratos en el período de potencia de punta, con un precio de venta promedio definido por la CDEEE, conforme va aumentando la penetración vehicular, van aumentado las pérdidas económicas, debido a las tarifas actuales.

\section{Conclusiones}

Con los resultados obtenidos se observó que la red actual de la República Dominicana de EDESUR debe iniciar los procesos de readecuación y actualización de las redes eléctricas de distribución con vistas al proceso de transición energética en la movilidad eléctrica que está comenzado a desarrollar, debido a que la demanda máxima en los transformadores de potencia en distribución, la caída de tensión en los nodos del sistema, las pérdidas técnicas y la intensidad de corriente que circula por los alimentadores de los circuitos son afectadas considerablemente por la inserción de los VE dentro de la matriz de transporte de la República Dominicana.

Desde el punto de vista económico y considerando las condiciones actuales de las redes de distribución, las pérdidas financieras de las empresas distribuidora de electricidad incrementarían a medida que aumenta la demanda de VE, esto provoca que el negocio de distribución sea menos rentable y demanda una reconstrucción que disminuyan las pérdidas técnicas asociadas. 
Con base en los diferentes escenarios analizados, la infraestructura de la red de distribución del Distrito Nacional de la República Dominicana no se encuentra en la capacidad de abastecer la demanda producida por los VE.

Se espera que este estudio sirva de punto de partida para investigaciones sobre la planificación de las redes de distribución, control y monitoreo de los VE.

\section{Agradecimientos}

A la empresa distribuidora de energía eléctrica del sur (EDESUR), por las informaciones y simulaciones realizadas en los softwares de flujo de potencia de los circuitos de distribución seleccionados.

A la Asociación de Movilidad Eléctrica Dominicana (ASOMOEDO), con sus informaciones sobre los perfiles de cargas de los vehículos de sus miembros se pudo asociar el comportamiento en la red de distribución de EDESUR en el Distrito Nacional, República Dominicana.

\section{Referencias}

Agudelo, P. A. (2018). Más de 700.000 vehículos eléctricos vendidos en el mundo a junio de 2018. Recuperado de https://www.vehiculoselectricos.co/mas-de-700-000-vehiculos-electricos-vendidos-en-elmundo-a-junio-de-2018/

Alaswad, A., Baroutaji, A., Achour, H., Carton, J., Al Makky, A., \& Olabi, A. G. (2016). Developments in fuel cell technologies in the transport sector. International Journal of Hydrogen Energy, 41(37), 16499-16508. doi: https://doi.org/10.1016/j.ijhydene.2016.03.164.

Anastasiadis, A. G., Kondylis, G. P., Polyzakis, A., \& Vokas, G. (2019). Effects of Increased Electric Vehicles into a Distribution Network. Energy Procedia, 157, 586-593. doi: https://doi.org/10.1016/j. egypro.2018.11.223

Aymeric, G., \& François, S. (2017). 7 - Case study for Chile: The electric vehicle penetration in Chile. In T. Muneer, M. L. Kolhe, \& A. Doyle (Eds.), Electric Vehicles: Prospects and Challenges (pp. 245-285): Elsevier. 
Baran, R., \& Legey, L. F. L. (2013). The introduction of electric vehicles in Brazil: Impacts on oil and electricity consumption. Technological Forecasting and Social Change, 80(5), 907-917. doi: https://doi. org/10.1016/j.techfore.2012.10.024

Bremermann, L. E., Matos, M., Lopes, J. A. P., \& Rosa, M. (2014). Electric vehicle models for evaluating the security of supply. Electric Power Systems Research, 111, 32-39. doi: https://doi.org/10.1016/j. epsr.2014.02.001

Cable, P. W. (2013). ACSR - Aluminum Conductor Steel Reinforced In (pp. 4).

Ceballos Delgado, J. E., Caicedo Bravo, E., \& Ospina Arango, Sandra. (2016). Una Propuesta Metodológica para Dimensionar el Impacto de los Vehículos Eléctricos sobre la Red Eléctrica. Ingeniería, 21, 22.

CNE, C. N. D. E. (2017). 1er. Seminario de Vehículos Eléctricos en República Dominicana. Recuperado de https://www.cne.gob.do/ noticia/kepco-y-cne-realizan-1er-seminario-de-vehiculos-electricos-en-rd/

Delgado, J., Faria, R., Moura, P., \& de Almeida, A. T. (2018). Impacts of plug-in electric vehicles in the portuguese electrical grid. Transportation Research Part D: Transport and Environment, 62, 372-385. doi: https://doi.org/10.1016/j.trd.2018.03.005

DGA, D. G. d. A. (2018). Vehículos eléctricos e hibridos exportados en República Dominicana periodo 2017-2018. (Caso 253 - 2018).

Díaz, K. (2017). Movilidad eléctrica, un desafío para el mercado dominicano, Artículo. Diario Libre. Recuperado de https://www.diariolibre. com/economia/movilidad-electrica-un-desafio-para-el-mercado-dominicano-FB7157052

EDESUR, D. (2018). Costos del Mercado Eléctrico Mayorista. In (pp. 2).

Electromaps. (2018). Puntos de recarga en Dominican Republic. Recuperado de https://www.electromaps.com/puntos-de-recarga/dominican-republic

EINozahy, M. S., \& Salama, M. M. A. (2014). Studying the feasibility of charging plug-in hybrid electric vehicles using photovoltaic electricity in residential distribution systems. Electric Power Systems Research, 110, 133-143. doi: https://doi.org/10.1016/j.epsr.2014.01.012 
Espinosa y Lara, R. (1990). Sistema de distribución. Monterrey, México: Limusa.

Ferrero, E., Alessandrini, S., \& Balanzino, A. (2016). Impact of the electric vehicles on the air pollution from a highway. Applied Energy, 169, 450-459. doi: https://doi.org/10.1016/j.apenergy.2016.01.098

Geerlings, H., \& Zuidwijk, R. (2017). Ports and Networks: Strategies, Operations and Perspectives: Taylor \& Francis.

Gode, H. a. P. (2016). Network planning for charging electric vehicles in Stockholm. (Project in Electrical Engineering), KTH Royal Institute of Technology, Sweden. Recuperado de https://kth.diva-portal.org/ smash/get/diva2:968635/FULLTEXT01.pdf.

Gómez, J., González, L., Vide, P., \& Gameiro, N. (2017). Impacto de las estaciones de carga para vehículo eléctrico en la curva de carga de la Ciudad de Cuenca. In Maskana (pp. 239-246): Congreso $\mathrm{I}+\mathrm{D}+$ ingeniería.

Jenn, A., Azevedo, I. L., \& Michalek, J. J. (2019). Alternative-fuel-vehicle policy interactions increase U.S. greenhouse gas emissions. Transportation Research Part A: Policy and Practice, 124, 396-407. doi: https://doi.org/10.1016/j.tra.2019.04.003

Mallig, N., Heilig, M., Weiss, C., Chlond, B., \& Vortisch, P. (2015). Modelling the Weekly Electricity Demand Caused by Electric Cars. Procedia Computer Science, 52, 444-451. doi: https://doi.org/10.1016/ j.procs.2015.05.012

Moon, H., Park, S. Y., Jeong, C., \& Lee, J. (2018). Forecasting electricity demand of electric vehicles by analyzing consumers' charging patterns. Transportation Research Part D: Transport and Environment, 62, 64-79. doi: https://doi.org/10.1016/j.trd.2018.02.009

Moreno Herrero, J. (2013). Impacto del vehículo eléctrico sobre las redes de distribución, (trabajo de grado ingenierio eléctrico). E.T.S.I. Minas, España.

Muñoz-Villamizar, A., Montoya-Torres, J. R., \& Faulin, J. (2017). Impact of the use of electric vehicles in collaborative urban transport networks: A case study. Transportation Research Part D: Transport and Environment, 50, 40-54. doi: https://doi.org/10.1016/j.trd.2016.10.018 
NREL, N. R. E. L. (2015). Energy Transition Initiative: Island Energy Snapshot - Dominican Republic. In (pp. 4).

Oficina Nacional de Estadísticas (ONE). (2010). Banco de microdatos de Censos, Encuestas y Registros Administrativos (BADECER) (Base de datos). Recuperado 16 de marzo http://redatam.one.gob. do/cgibin/RpWebEngine.exe/PortalAction?\&MODE=MAIN\&BASE=CPV2010\&MAIN=WebServerMain.inl

Riesz, J., Sotiriadis, C., Ambach, D., \& Donovan, S. (2016). Quantifying the costs of a rapid transition to electric vehicles. Applied Energy, 180, 287-300. doi: https://doi.org/10.1016/j.apenergy.2016.07.131.

Superintendencia de Electricidad (SIE). (2002). Regimen Tarifario Aplicable por las Empresas Distribuidoras. Recuperado de http://www.sie.gob. $\mathrm{do} /$ protecom/preguntas-frecuentes/item/16-dependiendo-de-mi-suministro-residencial-comercial-zona-franca-industrial-que-tarifa-debo-solicitar

SIE, S. d. e. (2015). Reglamento Diseño y Construcción para Redes Eléctricas de Distribución Aéreas. Santo Domingo Recuperado de http://www.sie.gob. do/images/sie-documentos-pdf/marco-legal/resoluciones-sie/2016/ RESOLUCION\%20SIE-029-2015-MEMI\%20-\%20REGL.\%20 DISE\%C3\%910\%20Y\%20CONSTRUCCION\%20REDES\%20 DIST.\%20AEREAS\%20-\%20III-A.pdf

SIE, S. d. e. (2016). Código eléctrico Nacional de la República Dominicana. (SIE-056-201-MEMI). Recuperado de http://sie.gob.do/images/ sie-documentos-pdf/marco-legal/resoluciones-sie/2016/RESOLUCION\%20SIE-056-2016-MEMI\%20\%20EMISION\%20 CODIGO\%20ELECTRICO\%20NACIONAL_merged2.pdf

Southwire. (2012). All Aluminum-Alloy Conductor. Bare. In (pp. 2). USA.

Transportation, I. C. o. C. (2015). Policies to Reduce Fuel Consumption, Air Pollution, and Carbon Emissions from Vehicles In G20 Nations. In (pp. 28).

Ul-Haq, A., Cecati, C., \& El-Saadany, E. (2018). Probabilistic modeling of electric vehicle charging pattern in a residential distribution network. Electric Power Systems Research, 157, 126-133. doi: https:// doi.org/10.1016/j.epsr.2017.12.005 
United Nations Economic Commission for Europe (UNECE). (2009). Climate change and sustainable transport. Recuperado de http:// www.unece. org $/$ ?id $=9890$

Vassileva, I., \& Campillo, J. (2017). Adoption barriers for electric vehicles: Experiences from early adopters in Sweden. Energy, 120, 632-641. doi: https://doi.org/10.1016/j.energy.2016.11.119

Wallington, T. J., Kaiser, E. W., \& Farrell, J. T. (2006). Automotive fuels and internal combustion engines: a chemical perspective. Chemical Society Reviews, 35(4), 335-347. doi: 10.1039/B410469M 